\title{
Canadian Urological Association-Canadian Urologic Oncology Group guideline on Metastatic castration-naive and castration-sensitive prostate cancer
}

\author{
Alan $\mathrm{So}^{1} ; \mathrm{Kim} \mathrm{Chi}^{2}$; Brita Danielson ${ }^{3}$; Neil Fleshner ${ }^{4} ;$ Anil Kapoor $^{5}$; Tamim Niazi ${ }^{6}$; Frederic \\ Pouliot $^{7}$; Ricardo Rendon ${ }^{8}$; Bobby Shayegan ${ }^{5}$; Srikala Sridhar ${ }^{9}$; Eric Vigneault ${ }^{10}$; Fred Saad ${ }^{11}$ \\ ${ }^{1}$ Department of Urologic Sciences, University of British Columbia, Vancouver, BC, Canada; ${ }^{2}$ Department of \\ Medicine, University of British Columbia, Vancouver, BC, Canada; ${ }^{3}$ Department of Oncology, Division of \\ Radiation Oncology, University of Alberta, Edmonton, AB, Canada; ${ }^{4}$ Division of Urology, University of Toronto, \\ Toronto, ON, Canada; ${ }^{5}$ Division of Urology, McMaster University, Hamilton, ON, Canada; ${ }^{6}$ Department of \\ Oncology, Division of Radiation Oncology, McGill University, Montreal QC, Canada; ${ }^{7}$ Division of Urology, \\ Department of Surgery, Université Laval, Quebec, QC, Canada; ${ }^{8}$ Department of Urology, Dalhousie University, \\ Halifax, NS, Canada; ${ }^{9}$ Division of Hematology and Medical Oncology, Princess Margaret Cancer Centre, University \\ Health Network, University of Toronto, Toronto, ON, Canada; ${ }^{10}$ Department of Radiation Oncology, CHUQ, \\ Université Laval, Quebec City, QC, Canada; ${ }^{11}$ Department of Surgery, Université de Montréal, Montreal, QC, \\ Canada
}

Cite as: Can Urol Assoc J 2019 December 5; Epub ahead of print. http://dx.doi.org/10.5489/cuaj.6384

Published online December 5, 2019

$* * *$

\section{Introduction}

Metastatic prostate cancer remains an incurable disease. In Canada, approximately $8 \%$ of men with prostate cancer are diagnosed de novo with metastatic disease, and, in 2018, roughly 1200 men were diagnosed with de novo metastatic prostate cancer (PC) (1). The mainstay of treatment for de novo metastatic PC is androgen deprivation therapy (ADT) which is initially effective in almost all patients. Progression is inevitable however, heralded by a rise in PSA, increasing disease burden and/or worsening symptoms, a disease state called metastatic castration resistant prostate cancer (mCRPC).

Men with de novo metastatic PC have a poor prognosis with an estimated median overall survival of approximately 3-4 years (2). This has only improved slightly even in the advent of improved management of mCRPC $(2,3)$. Compared to PC that develops metastases after diagnosis, de novo metastatic $\mathrm{PC}$ has been shown to have a worse prognosis $(4,5)$. Recent practice changing trials have shed light on new directions to improve survival in men with metastatic castration-naïve /castration sensitive PC (mCNPC/mCSPC), and include both systemic therapies and treatment of the primary cancer.

The Canadian Urologic Oncology Group (CUOG) in collaboration with the CUA sought to provide management guidelines to optimize the treatment of $\mathrm{mCNPC} / \mathrm{mCSPC}$ patients. 


\section{Methods}

EmBASE and Medline databases were accessed to identify all relevant articles focused on mCNPC or mCSPC between January 2000 and August 2019 with the following key words strategy: "prostate cancer", "hormone sensitive", "castration naïve", "castration sensitive", "androgen deprivation", "chemotherapy", "androgen receptor-axis targeted therapy", and "metastatic." An expert panel comprised of urologists, medical oncologists and radiation oncologists with significant experience managing $\mathrm{mCNPC} / \mathrm{mCSPC}$ was utilized to develop the recommendations. Guidelines were developed by consensus among the panel. Levels of evidence and grades of recommendation employ the WHO modified Oxford Center for Evidence-Based Medicine grading system(6). Based on a modified GRADE methodology, the strength of each recommendation is represented by the words STRONG or WEAK(6). Wherever Level 1 evidence is lacking, the guideline attempts to provide expert opinion to aid in the management of patients.

\section{Indications for staging in prostate cancer}

For newly diagnosed PC, staging with CT scan of the abdomen and pelvis and bone scan (99mTc-MDP) should be performed for men with any high risk features: PSA>20 ng/mL, Gleason score $>\mathbf{7}$, clinical stage $\mathbf{T 3}$ or greater (Level of evidence 3, Strong recommendation).

Conventional imaging to stage PC includes, bone scintigraphy using technetium-

99mmethylene diphosphonate (99mTc-MDP) to assess for bone metastases and abdominopelvic CT imaging to assess for lymphadenopathy and visceral metastases. In patients with high risk disease, CT imaging of the chest may also be considered as lung metastases are the most common site of visceral metastases(7).

Novel diagnostic imaging to stage PC including choline-based PET/CT, fluciclovine PET/CT and PSMA-targeted PET/CT, appear to improve the sensitivity and specificity of conventional imaging; however, these tests are not universally available Canada, their clinical utility is not clear and they are still considered investigational by Health Canada. Most importantly, all of the phase 3 trials in $\mathrm{mCNPC} / \mathrm{mCSPC}$ utilized conventional imaging for staging and risk determination, and conclusion were based on these. Novel imaging remains investigational.

\section{Assessment of prognosis}

Patients diagnosed with metastatic PC should be classified as high volume/high risk or low volume/low risk based on conventional imaging and prostate cancer biopsy for prognostication (Level of evidence 2 , Weak recommendation).

Utilizing data from two large mCNPC/mCSPC trials, SWOG8894 and GETUG15, possible prognostic features suggestive of worse prognosis have been identified and include: appendicular disease (defined as bone lesions in the chest, skull and/or extremities), worse performance status, prostate specific antigen greater than 65, Gleason score of 8 or greater, high 
alkaline phosphatase (ALP), high pain intensity, anemia, and elevated LDH $(4,5)$. Data from SWOG8894 suggests that appendicular disease is the strongest predictor prognosis whereas GETUG15 suggested, based on univariate analysis, that ALP is the strongest predictor of prognosis $(4,5)$.

Recent clinical trials of $\mathrm{mCNPC} / \mathrm{mCSPC}$ patients have used different pragmatic prognostic factors to stratify prognosis. The CHAARTED trial classified PC based on volume of disease. 'High volume' was defined by the presence of visceral metastases or $\geq 4$ bone lesions with $\geq 1$ beyond the vertebral bodies and pelvis, and 'low volume' was defined as all other $\mathrm{mCNPC} / \mathrm{mCSPC}$ (8). The LATITUDE trial classified 'high risk' patients based on three different criteria: visceral metastases, $\geq 3$ bony metastases or Gleason score $\geq 8$; high risk was defined as having 2 or more of these criteria whereas low risk was defined having less than 2 (9). Interestingly, a comparative study of the classification of each of these trials showed an overall discordance of $18.2 \%$ between the CHAARTED and LATITUDE criterion; however, it appears that disease burden (defined radiologically or by PSA) and high grade tumors portend a worse prognosis (10).

\section{Androgen-deprivation therapy}

ADT should be started on men newly diagnosed with metastatic PC (Level of evidence 1, Strong recommendation).

\section{Continuous ADT is the standard of care for metastatic PC while intermittent may be considered in select patients.}

Androgen receptor signaling plays a key role in the progression of $\mathrm{PC}$, and thus de novo mCNPC remains highly driven by testosterone. Hence, the primary step in the management of mCNPC, which remains the backbone of treatment for all men with metastatic PC until death, is androgen deprivation therapy (ADT). ADT can be achieved by surgical castration (orchiectomy) or pharmacologically with agents that inhibit Leydig cell production of testosterone (GnRH agonists or GnRH antagonists). The optimal timing of androgen deprivation has been the subject of many trials with two large recent systematic reviews suggesting early treatment is associated with improved overall and cancer-specific survival and decreases the rate of skeletal events compared to deferred treatment $(11,12)$. More importantly, the early treatment of mCNPC with ADT is required if other systemic treatment such as docetaxel or androgen receptor axis inhibitors are used.

ADT is associated with increased side effects, and may increase the risk of cardiovascular events. Intermittent androgen suppression (IAS) that cycles androgen deprivation treatment based on PSA values has been shown to improve quality of life; however, continuous ADT should be utilized in mCNPC and IAS only used as an exception in select patients with close follow-up $(13,14)$. As well, combined treatment of mCNPC with any systemic therapy requires continuous ADT. 


\section{Local therapy: Treatment of the primary cancer in mCNPC}

\section{Patients with low volume metastatic disease burden should be considered for external beam radiation to the prostate (Level of evidence 2, Strong recommendation).}

Treatment of the primary PC has theoretical benefits, including reducing local side effects that may occur due to disease progression during MCRPC and well as removing the cancer that could be source of cytokines and growth factors that may induce disease progression.

Two recent randomized trials assessed the impact of external beam radiation therapy (EBRT) in mCNPC. The HORRAD trial randomized 432 men with mCNPC and PSA $>20 \mathrm{ng} / \mathrm{mL}$ to receive EBRT of the prostate with ADT or ADT alone. The initial prescribed dose was $70 \mathrm{~Gy}$ in 35 fractions of $2 \mathrm{~Gy}$, during an overall treatment time of 7 weeks. During the study period, an optional schedule was added that was considered biologically equivalent and consisted of a dose schedule of $57.76 \mathrm{~Gy}$ in 19 fractions of $3.04 \mathrm{~Gy}$, three times a week for 6 weeks. The median PSA was $142 \mathrm{ng} / \mathrm{ml}$ and $67 \%$ of patients had more than five bone metastases. No significant difference was found in overall survival (OS) (hazard ratio (HR), 0.90; 95\% CI: 0.70-1.14; $\mathrm{p}=$ $0.4)$, but there was a benefit to median time to PSA progression in the radiotherapy group $(15 \mathrm{~m}$ vs $12 \mathrm{~m}$, crude HR, 0.78 ; 95\% CI: $0.63-0.97 ; \mathrm{p}=0.02$ ). Subgroup analysis showed that $\mathrm{mCNPC}$ with $<5$ metastases (HR, 0.90, 95\% CI: 0.70-1.14, p=NS) and no bony pain (HR, $0.83,95 \% \mathrm{CI}$ : 0.69-1.14, $\mathrm{p}=\mathrm{NS}$ ) appeared to have the most impact of EBRT.

The STAMPEDE trial, also known as MRC PR08, is a multi-arm multi-stage (MAMS) randomized trial recruiting in the UK and Switzerland. It aims to evaluate multiple therapeutic strategies in the management of high-risk locally advanced and mCNPC compared to standard of care (androgen deprivation only). In the EBRT component of the study, the trial randomized 2061 men with mCNPC to either EBRT and ADT or ADT alone(15). The median PSA was 97 $\mathrm{ng} / \mathrm{mL}$, and $819(40 \%)$ men had low metastatic burden based on CHAARTED criteria and 1664 $(81 \%)$ had no pain $(8,15)$. EBRT was given as one of two schedules: either $36 \mathrm{~Gy}$ in six consecutive weekly fractions of $6 \mathrm{~Gy}$, or $55 \mathrm{~Gy}$ in 20 daily fractions of 2.75 Gy over 4 weeks. Subgroup analyses were pre-specified for baseline metastatic burden (low vs high).

Similar to the HORRAD trial, EBRT improved failure-free survival (FFS) (HR, 0.76, $95 \%$ CI $0.68-0.84 ; \mathrm{p}<0.0001)$ but not OS $(0.92,0.80-1.06 ; \mathrm{p}=0.266)$. Subgroup analysis by metastatic burden showed FFS was improved in both low and high metastatic burden (low metastatic burden, HR, 0.59, 95\% CI 0.49-0.72; $\mathrm{p}<0.0001$ and metastatic burden, interaction $\mathrm{p}=0.002 ; \mathrm{HR}, 0.88,95 \% \mathrm{CI} 0.77-1 \cdot 01 ; \mathrm{p}=0.059$ ). OS was improved in patients with low metastatic burden at baseline who were allocated EBRT (HR, 0.68, 95\% CI 0.52-0.90; $\mathrm{p}=0.007$ ) whereas in patients with a high metastatic burden, there was no impact on OS (HR, 1.07, 95\% CI $0.90-1.28 ; \mathrm{p}=0.420$ ).

Although both trials showed negative impact of EBRT in unselected men in mCNPC, both HORRAD and STAMPEDE reveal the benefits of local therapy in those with low burden disease. A recent STOPCAP meta-analysis combining data from the trials confirm the benefits of 
EBRT in men with fewer than five bone metastases (16). This meta-analysis showed that there was $7 \%$ improvement in 3-yr survival in men with fewer than four bone metastases.

Radical prostatectomy in mCNPC should only be performed in a clinical trial setting (Expert opinion, Strong recommendation).

Currently, there is limited evidence showing the benefit of radical prostatectomy in mCNPC. However, the results from HORRAD and STAMPEDE imply that there may also be certain men with mCNPC that may benefit from surgical extirpation. There are many clinical trials currently assessing this question, including TRoMBONE (Testing radical prostatectomy in men with PC and oligometastases to the bone: a randomized controlled feasibility trial)(17), SWOG1802 (Standard systemic therapy with or without definitive treatment in treating participants with metastatic PC- https://www.swog.org/clinical-trials/s1802) and GRAMPP/AUO -AP-75/13 (Impact of radical prostatectomy as primary treatment in patients with PC with limited bone metastases)(18). Until the results of these trials clarify the impact of radical prostatectomy in mCNPC and more importantly which patients that would benefit the most, surgery of the primary is not recommended in patients with metastatic PC.

Systemic therapies: Chemotherapy, abiraterone acetate, enzalutamide, and apalutamide

Docetaxel ( $75 \mathrm{mg} / \mathrm{m}^{2}$ every 3 weeks for six cycles) plus ADT is an option for men with $\mathrm{mCNPC} / \mathrm{mCSPC}$ and having good performance status and high volume metastatic disease defined as: presence of visceral metastases, or four or more bone lesions with at least one beyond the vertebral bodies and pelvis (Level 1, Strong recommendation).

\section{Docetaxel plus ADT may also be an option in $\mathbf{m C N P C / m C S P C}$ and having good performance status with low volume disease (Level 2, Weak recommendation).}

Consideration of "high risk" mCNPC/mCSPC patients (defined as at least two of: Gleason score of 8-10, visceral metastases and 3 or more bone metastases) and good performance status can also be considered for docetaxel chemotherapy (Level 1, Strong recommendation).

Docetaxel, a taxane derivative that binds to tubulin that inhibits mitosis and tumour proliferation, was the initial chemotherapeutic agent that improved survival in men mCRPC (19). Three different large randomized trials assessed the impact of introducing docetaxel in mCNPC/mCSPC: CHAARTED, STAMPEDE, and GETUG-AFU 15(8, 20, 21). The CHAARTED trial randomized 790 with $\mathrm{mCNPC} / \mathrm{mCSPC}$ patients to ADT plus docetaxel (75 $\mathrm{mg} / \mathrm{m}^{2}$ every 3 weeks for six cycles) or ADT alone (8). Within this trial, 35\% (277pts) had low volume metastases and 65\% (513pts) had high volume metastases (high volume of metastases was defined by the presence of visceral metastases or four or more bone lesions with at least one beyond the vertebral bodies and pelvis). Overall, the median OS was 13.6 months longer with ADT plus docetaxel than with ADT alone (57.6 months vs. 44.0 months; HR, 0.61 ; 95\% CI 0.47- 
0.80; $\mathrm{P}<0.001)$. Subgroup analysis showed that OS benefits of combination there were maintained in the high volume $\mathrm{mCNPC} / \mathrm{mCSPC}(\mathrm{n}=513$, HR, 0.63 (95\% CI, 0.50-0.79) $\mathrm{P}<0.001)$ whereas survival benefits were lost in low volume disease $(\mathrm{n}=277, \mathrm{HR}, 1.04$ (95\% CI, $0.70-1.55) \mathrm{P}=0.86)(22)$.

The GETUG-AFU15 trial randomized $385 \mathrm{mCNPC} / \mathrm{mCSPC}$ patients to receive ADT plus docetaxel or ADT alone (21). Although the dosage of docetaxel was the same as in CHAARTED, patients were allowed to receive up to nine cycles compared to the six cycles in CHAARTED. There was no survival difference between the groups (58.9 months in the combined group vs 54.2 months in the ADT alone group, HR, 1.01, 95\% CI 0.75-1.36). The differences in the outcomes of the two studies is likely due to the differences in the burden of disease in the two studies. Although $65 \%$ of patients in CHAARTED had high volume metastases, less than $25 \%$ of the patients had low-volume disease. An unplanned post-hoc analysis of the high volume cohort of GETUG-AFU 15 showed a non-significant trend toward improved OS in this cohort (39.8 months vs 35.1 months, HR, 0.78, 95\% CI, 0.56-1.09) (23). A recent pooled analysis of both studies confirm the benefit of combined docetaxel and ADT in high-volume disease and lack of benefit on low-volume metastatic burden (24).

The third trial to assess the impact of docetaxel in mCNPC/mCSPC was the docetaxel component of the STAMPEDE trial(20). Unlike CHAARTED and GETUG-AFU15 trials, patients with high-risk non-metastatic PC were included. Eligible patients included: newly diagnosed metastatic, node positive, or high-risk locally advanced (with high risk features defined as at least two of: T3/4, Gleason score of 8-10, and PSA $\geq 40 \mathrm{ng} / \mathrm{mL}$ ); or previously treated with radical surgery and/or radiotherapy with high-risk features. Of the $2962 \mathrm{pts}$ randomized, 1817 (61\%) men had bony metastases and 592 pts received only ADT and six cycles of docetaxel ( $75 \mathrm{mg} / \mathrm{m}^{2}$ every 3 weeks for six cycles). The combination of ADT and docetaxel had a survival advantage compared to ADT alone (HR, 0.78, 95\% CI, 0.66-0.93; $\mathrm{p}=0.006$ ). Although patients were not classified having high or low volume metastases, only patients with metastatic disease had evidence of benefit with ADT and docetaxel (HR, 0.76, 95\% CI, 0.62-0.92; $\mathrm{p}=0.005)$.

A recent post-hoc non-prespecified analysis of STAMPEDE was published(25). Metastatic burden was assessable in only $76 \%$ of patients for the analysis (830 of 1086 patients) and 362 (44\%) had low and 468 (56\%) high metastatic burden. Although overall survival was neither statistically significant in low burden nor in high burden disease (HR, 0.76, 95\% CI, 0.54-1.07; $\mathrm{p}=0.107$ vs HR, 0.81, CI, 0.64-1.02, p=0.064), the authors found no evidence of heterogeneity of docetaxel effect between metastatic burden sub-groups (interaction $\mathrm{p}=0.827$ ). The authors concluded that upfront docetaxel is considered for mCNPC/mCSPC patients regardless of metastatic burden. This retrospective analysis contradicts the results of CHAARTED, but the authors point out that this may be due to the larger number of de novo $\mathrm{mCNPC} / \mathrm{mCSPC}(\mathrm{n}=362)$ in the low burden group compared to the low burden group in the CHAARTED trial $(\mathrm{n}<160)$. 
A recent meta-analysis of CHAARTED, GETUG-AFU15, and STAMPEDE confirms the benefit of addition of docetaxel to ADT in $\mathrm{mCNPC} / \mathrm{mCSPC}$ (HR, 0.77, 95\% CI, 0.68-0.87, $\mathrm{p}<0.0001)$. The authors of the meta-analysis show that this translates to an absolute improvement in 4-year survival of $9 \%$.

\title{
Abiraterone acetate $(1000 \mathrm{mg}$ daily) with prednisone (5mg daily) plus ADT is an option for mCNPC patients with at least two of the three: (Gleason score of $\geq 8$, presence of three or more lesions on bone scan, or presence of measurable visceral metastasis) (Level of evidence 1, Strong recommendation).
}

\begin{abstract}
Abiraterone acetate (1000mg daily) with prednisone (5mg daily) plus ADT may be considered for patients with low volume mCNPC (Level of evidence 3, Weak recommendation).
\end{abstract}

Abiraterone acetate is a prodrug of abiraterone which is a CYP17A1 inhibitor; CYP17A1 is expressed in and is required for androgen biosynthesis. Abiraterone acetate, when combined with prednisone, was initially shown to improve survival in mCRPC, both prior to and after docetaxel treatment $(26,27)$. Two trials, LATITUDE and STAMPEDE, assessed the impact of abiraterone in $\mathrm{mCNPC} / \mathrm{mCSPC}(9,28,29)$. In the LATITUDE trial, 1199 patients were randomly assigned to either the abiraterone acetate $(1000 \mathrm{mg})$ plus prednisone $(5 \mathrm{mg})$ once daily orally. Eligible patients included mCNPC with at least two of three high-risk features (Gleason score of $\geq 8$, presence of three or more lesions on bone scan, or presence of measurable visceral metastasis except lymph node metastasis). Updated OS data with median follow-up of 51.8 months showed that OS was significantly longer in the abiraterone acetate plus prednisone group (median 53.3 months [95\% CI 48.2-not reached]) than in the placebo group (median 36.5 months [95\% CI 33.5-40.0]), with a hazard ratio of $0.66(95 \%$ CI $0.56-0.78$; $p<0 \cdot 0001)$. A posthoc exploratory analysis of the impact of disease burden showed that OS was improved only in high-volume disease $(n=487$ in the abiraterone acetate plus prednisone and ADT and 468 in the ADT only group, HR 0.62 (95\% CI, 0.52-0.74; p<0.0001); however, only few patients had lowvolume disease in this study $(\mathrm{n}=110$ in the abiraterone acetate plus prednisone and ADT and $\mathrm{n}=133$ in the ADT only group, (HR 0.72, 95\% CI 0.47-1.10; $\mathrm{p}=0 \cdot 1242$ ).

In the abiraterone component of the STAMPEDE trial, the efficacy of abiraterone acetate and prednisolone was assessed in men with mCNPC (28). In this study, 1917 mCNPC patients were enrolled with: newly diagnosed and metastatic, node-positive, or high-risk locally advanced (with at least two of following: cT3 or cT4, a Gleason score of 8 to 10 , or PSA level $\geq 40 \mathrm{ng} / \mathrm{mL}$ ) or disease that was previously treated with radical surgery or radiotherapy and was now relapsing with high-risk features (PSA $>4 \mathrm{ng} / \mathrm{mL}$ with a doubling time of $<6$ months, a PSA level $>20$ $\mathrm{ng} / \mathrm{mL}$, nodal or metastatic relapse). Men were randomized to receive abiraterone acetate (1000mg daily) plus prednisolone (5mg) plus ADT or ADT alone. 52\% of the patients had metastatic disease, $20 \%$ had node-positive or node-indeterminate non-metastatic disease, and 
$28 \%$ had node-negative, non-metastatic disease; $95 \%$ had newly diagnosed disease. In a subgroup analysis, the overall survival benefit was seen in PC patients with metastatic disease (HR $0.61,95 \%$ CI 0.49-0.75) but not those with non-metastatic high risk patients (HR 0.75, 95\% CI 0.48-1.18) (28). The impact of volume tumor burden was not reported.

A recent unplanned post-hoc analysis of 759 evaluable patients with bone metastases in above STAMPEDE trial were reclassified using CHAARTED "high or low volume" criterion or LATITUDE "high or low risk" criterion (30). Men with mCNPC had OS benefit with the addition of abiraterone acetate and prednisone to ADT irrespective of risk stratification for "risk" or "volume". Using CHAARTED criteria, low volume HR was 0.66 (95\% CI, 0.44-0.98) and high volume HR was 0.54 (95\% CI 0.41-0.70); using the LATITUDE criteria, low risk HR was 0.64 (95\% CI, 0.42-0.97) and high risk HR was 0.60 (95\% CI, 0.46-0.78). Although these results are intriguing, the retrospective nature of the reclassification of risk and tumor volume is a significant limitation and thus the results can only be considered hypothesis generating.

Enzalutamide $(160 \mathrm{mg} /$ day) is a treatment option for $\mathrm{mCNPC} / \mathrm{mCSPC}$ regardless of volume of disease (Level of evidence 1, Strong recommendation).

\section{Enzalutamide should not be used in combination (concurrent use) with docetaxel to treat mCNPC/mCSPC (Level of evidence 2, Strong recommendation).}

\section{Enzalutamide may be considered in MCSPC patients previously treated with docetaxel} chemotherapy (sequential use) (Level of evidence 1, Weak recommendation).

Enzalutamide binds to the androgen receptor (AR) and inhibits the AR nuclear translocation and interaction with DNA. Suppression of the AR with enzalutamide was initially shown to improve survival in docetaxel naïve or treated $\operatorname{mCRPC}(31,32)$. Two recent studies assessed the role of enzalutamide in mCNPC: ARCHES and ENZAMET $(33,34)$. The ARCHES trial randomized $1150 \mathrm{mCNPC} / \mathrm{mCSPC}$ patients to either enzalutamide (160mg/day) plus ADT or placebo plus ADT. The primary endpoint was radiologic progression free survival (rPFS), defined as the time from randomization to the first objective evidence of radiographic disease progression or death. The combination of enzalutamide plus ADT improved rPFS compared to placebo-ADT (hazard ratio $=0.39 ; 95 \% \mathrm{CI}, 0.30-0.50 ; \mathrm{P}=0.001$; median not reached $\mathrm{v} 19.0$ months). Due to the immaturity of the study and the median duration of OS, median OS was not reached in either arm and no survival differences were observed between the two arms. Interestingly, prior docetaxel of up to six cycles was allowed, and 18\% (205) men received at least one dose of docetaxel prior to randomization; subgroup analysis showed that rPFS benefit was seen in both, chemotherapy-treated and chemotherapy-naïve patients. As well, although 35\% (405 pts) of men were low volume based on CHAARTED criteria, benefit in rPFS with enzalutamide treated was seen regardless of volume of disease. 
ENZAMET was an open -label clinical trial that randomized 1125men with $\mathrm{mCNPC} / \mathrm{mCSPC}$ to receive ADT and enzalutamide daily $(160 \mathrm{mg}$ ) or a nonsteroidal antiandrogen (NSAA: bicalutamide, nilutamide, or flutamide) with a primary endpoint of OS. There was an OS benefit in the enzalutamide plus ADT arm compared to NSAA (hazard ratio = $0.67 ; 95 \%$ CI, 0.52 - 0.86; P = 0.002). Kaplan-Meier estimates of overall survival at 3 years were $80 \%$ in the enzalutamide group and $72 \%$ in the NSAA arm. Unlike ARCHES, concurrent use of docetaxel was allowed and decision to treat with chemotherapy was at the discretion of the investigator. Use of chemotherapy was well balanced between the two arms (45\% of those receiving enzalutamide and $44 \%$ of those receiving a NSAA planned for early docetaxel use). In a subgroup analysis, the benefits of enzalutamide on OS appeared only in the group without planned early docetaxel use (concurrent docetaxel: HR, 0.9 with a 95\% CI, 0.62-1.31, and no concurrent docetaxel: HR, 0.8 with a $95 \%$ CI, 0.59-1.07). Although the authors state that the study is underpowered and data is too immature to specifically answer whether or not combination docetaxel and enzalutamide is beneficial in $\mathrm{mCNPC} / \mathrm{mCSPC}$, these results show that this combination should not be used until further evidence is shown for its benefits.

\section{Apalutamide (240mg) is a treatment option for men with $\mathrm{mCNPC} / \mathrm{mCSPC}$ regardless of} volume of disease (Level of evidence 1, Strong recommendation).

Apalutamide inhibits the AR by preventing its nuclear translocation and DNA binding. The first large randomized clinical trial assessing apalutamide in $\mathrm{mCNPC} / \mathrm{mCSPC}$ was the TITAN trial, which randomized 1052 men with $\mathrm{mCNPC} / \mathrm{mCSPC}$ (any) to receive apalutamide (240mg once daily) plus ADT or ADT alone. As well, 10.7\% received previous docetaxel therapy and $37.3 \%$ had low-volume disease. With a median of 22.7 months of follow-up, rPFS at 24 months was $68.2 \%$ in the apalutamide group and $47.5 \%$ in the placebo group (HR, 0.48; $95 \%$ CI, 0.39 - 0.60, P<0.001). Benefit with apalutamide in rPFS was seen regardless of prior chemotherapy use or disease burden. Overall survival at 24 months was also greater with apalutamide than with placebo $(82.4 \%$ in the apalutamide group vs. $73.5 \%$ in the placebo group; $\mathrm{HR}, 0.67 ; 95 \% \mathrm{CI}, 0.51$ to $0.89 ; \mathrm{P}=0.005)(35)$. Benefit with apalutamide in OS was seen regardless of disease burden.

\section{Prevention of osteoporosis}

All men with mCNPC/mCSPC treated with ADT should be assessed for fracture risk. All men treated with ADT require vitamin D supplementation (800-1200IU daily) and calcium supplementation $(800 \mathrm{mg}-1000 \mathrm{mg}$ total intake daily). Those at high risk of fractures should be treated (zoledronic acid $5 \mathrm{mg}$ once a year, alendronate $70 \mathrm{mg}$ weekly, denosumab $60 \mathrm{mg}$ every 6 months).

Due to the evolution of combined therapy with ADT to treat mCNPC, the survival of men with de novo $\mathrm{PC}$ is increasing and length of time bone is exposed to the effects of ADT is increasing. As such, these men are at risk of significant bone loss and are at risk of osteoporosis and fragility fractures. Bone loss occurs quickly while on ADT and within one year men can lose 
up to $10 \%$ of their bone mineral density (BMD)(36-38). Men with mCNPC initiating ADT should have baseline BMD with dual-energy x-ray absorptiometry (DXA) as well as utilization of fracture risk calculators such as FRAX(39). DXA should be performed at least every two years and more often in untreated patients at high risk or if there is a history of osteoporosis/osteopenia.

Men with mCNPC/mCSPC treated with ADT should be encouraged to take vitamin D (1000IU daily) and total calcium intake of at $800 \mathrm{mg}-1000 \mathrm{mg}$ daily. Specific lifestyle changes including: smoking cessation, reduction in alcohol and caffeine intake and increase weightbearing exercises. If DXA scanning shows any evidence of osteopenia (T-score of $\langle-1$ and $\rangle$ 2.5) or osteoporosis (T-score of less than -2.5) men should be started a bone targeted therapy to improve BMD and reduce the risk of fragility fractures (zoledronic acid $5 \mathrm{mg}$ once a year, alendronate $70 \mathrm{mg}$ weekly, denosumab $60 \mathrm{mg}$ every 6 months $)(37,38,40)$. Bone targeted therapy at these doses are much lower than those to prevent SREs in MCRPC and therefore, are associated with significantly reduced side effects; incidence of clinically significant hypocalcemia and osteonecrosis of the jaw is rare using denosumab or zoledronic acid with these lower doses. $(41,42)$

\section{Treatment of oligo-metastatic disease}

There is evolving evidence of the role of radiation to asymptomatic distant metastases, especially in low burden "oligometastatic" disease. Currently, there is limited data to provide general recommendations, however, consideration in a multi-disciplinary setting would provide the best setting to determine optimal management consideration case-by-case.

\section{Multidisciplinary consultation}

\section{Men with $\mathbf{m C N P C / m C S P C}$ should be assessed in a multidisciplinary manner whenever possible (Level of evidence 3, strong recommendation).}

Timing of initiation and choosing the optimal systemic therapy from a multitude of options requires careful consideration of multitude of different clinical factors such as eligibility of chemotherapy, side effect profile of medications, disease burden, symptoms, and presence of visceral metastases. Since treatment may require a multifaceted approach, opinions from urology, medical oncology and radiation oncology may be required to provide optimal care or $\mathrm{mCNPC} / \mathrm{mCSPC}$ patients. Additionally, as mCNPC /mCSPCcontinues to be an incurable

disease, strong consideration should be given to inclusion of patients in clinical trials.

\section{Conclusions}

The last 5 years has seen a significant growth of life extending therapies for patients that has changed the landscape of treatment for $\mathrm{mCNPC} / \mathrm{mCSPC}$. These treatments range from treatment of the primary cancer with external beam radiation to chemotherapy. All men with mCNPC should be considered for treatments that are combined with ADT; those with high risk/ high 
volume disease should be given systemic therapy and those with low risk/ low volume should be strongly considered for prostate radiation therapy and / or systemic therapy.

\section{Conflicts of interest:}

Dr. So has been an advisory board member for Abbvie, Amgen, Astellas, Bayer, Janssen, Ferring, and TerSera; and has participated in clinical trials supported by Astellas, Ferring, and Janssen. Dr. Chi has received honoraria from Astellas, Bayer, Janssen, and Sanofi; and has participated in clinical trials supported by Astellas, AstraZeneca, Bayer, Eli Lilly, Essa, Janssen, Merck, Novartis, Pfizer, Roche, and Sanofi.Dr. Danielson has received advisory board honoraria and speaker fees from Amgen, Astellas, Bayer, and Janssen. Dr. Fleshner has been a consultant or advisory board member for Abbvie, Amgen, Astellas, Bayer, Ferring, Hybridyne Health, Janssen, and Sanofi; and has participated in clinical trials supported by Astellas, Bavarian Nordic, Bayer, Ferring, Janssen, Medivalion, Nucleix, Progenics Pharmaceutical, Sanofi, and Spectracure AB. Dr. Kapoor has been an advisory board member for BMS, Eisai, Ipsen, Merck, Novartis, Pfizer, and Roche; a speakers' bureau member for Eisai, Ipsen, Novartis, and Roche; and has received grants/honoraria from BMS, Eisai, Ipsen, Merck, Novartis, Pfizer, and Roche. Dr. Niazi has received research grants and honoraria from Abbvie, Amgen, Astellas, Astra Zeneca, Bayer, Janssen, and Sanofi; and has participated in clinical trials supported by Astellas, Ferring, Janssen, and Sanofi. Dr. Pouliot has been an advisory board member for Amgen, Astellas, Bayer, and Janssen; has received payment from Abbott, Amgen, Astellas, Astra Zeneca, Bayer, Ferring, Janssen, and Sanofi; has received grants from Astra Zeneca and Sanofi; and has participated in clinical trials supported by Astellas, Bayer, Ferring, and Janssen. Dr. Rendon has been an advisory board and speakers' bureau member for and has received honoraria from Abbvie, Amgen, Astellas, Astra Zeneca, Bayer, Ferring, Jansen, and Sanofi. Dr. Shayegan has been an advisory board member for Astellas, Bayer, and Janssen; and has received a research grant from Janssen. Dr. Shayegan has been an advisory board member for Astellas, Bayer, and Janssen; and has received a research grant from Janssen. Dr. Sridhar has been an advisory board member for Astellas, AstraZeneca, Bayer, Janssen, Merck, and Roche; and has participated in several pharma-supported clinical trials. Dr. Vigneault has been an advisory board member for Abbvie, Bayer, Ferring, and Sanofi. Dr. Saad has been an advisory board member for and has received payment/honoraria from Abbvie, Amgen, Astellas, Bayer, Janssen, and Sanofi; and has participated in clinical trials supported by Amgen, Astellas, Bayer, Janssen, and Sanofi. 


\section{References}

1. Committee CCSA. Canadian Cancer Statistics 2018. Canadian Cancer Society. 2019.

2. Glass TR, Tangen CM, Crawford ED, Thompson I. Metastatic carcinoma of the prostate: identifying prognostic groups using recursive partitioning. J Urol. 2003;169(1):164-9.

3. Gravis G, Boher JM, Fizazi K, Joly F, Priou F, Marino P, et al. Prognostic Factors for Survival in Noncastrate Metastatic Prostate Cancer: Validation of the Glass Model and Development of a Novel Simplified Prognostic Model. European urology. 2015;68(2):196-204.

4. Frees S, Akamatsu S, Bidnur S, Khalaf D, Chavez-Munoz C, Struss W, et al. The impact of time to metastasis on overall survival in patients with prostate cancer. World J Urol. 2018;36(7):1039-46.

5. Mosillo C, Iacovelli R, Ciccarese C, Fantinel E, Bimbatti D, Brunelli M, et al. De novo metastatic castration sensitive prostate cancer: State of art and future perspectives. Cancer Treat Rev. 2018;70:67-74.

6. Guyatt GH, Oxman AD, Vist GE, Kunz R, Falck-Ytter Y, Alonso-Coello P, et al. GRADE: an emerging consensus on rating quality of evidence and strength of recommendations. BMJ. 2008;336(7650):924-6.

7. Bubendorf L, Schopfer A, Wagner U, Sauter G, Moch H, Willi N, et al. Metastatic patterns of prostate cancer: an autopsy study of 1,589 patients. Hum Pathol. 2000;31(5):578-83.

8. Sweeney CJ, Chen YH, Carducci M, Liu G, Jarrard DF, Eisenberger M, et al. Chemohormonal Therapy in Metastatic Hormone-Sensitive Prostate Cancer. The New England journal of medicine. 2015;373(8):737-46.

9. Fizazi K, Tran N, Fein L, Matsubara N, Rodriguez-Antolin A, Alekseev BY, et al. Abiraterone acetate plus prednisone in patients with newly diagnosed high-risk metastatic castration-sensitive prostate cancer (LATITUDE): final overall survival analysis of a randomised, double-blind, phase 3 trial. Lancet Oncol. 2019;20(5):686-700.

10. Iacovelli R, Ciccarese C, Schinzari G, Maiorano BA, Rossi E, Pierconti F, et al. Going towards a precise definition of the therapeutic management of de-novo metastatic castration sensitive prostate cancer patients: How prognostic classification impact treatment decisions. Crit Rev Oncol Hematol. 2019;139:83-6.

11. Nair B, Wilt T, MacDonald R, Rutks I. Early versus deferred androgen suppression in the treatment of advanced prostatic cancer. Cochrane Database Syst Rev. 2002(1):CD003506.

12. Kunath F, Goebell PJ, Wullich B, Sikic D, Kahlmeyer A. Timing of androgen deprivation monotherapy and combined treatments in castration-sensitive and castration-resistant prostate cancer: a narrative review. World J Urol. 2019. 
13. Niraula S, Le LW, Tannock IF. Treatment of prostate cancer with intermittent versus continuous androgen deprivation: a systematic review of randomized trials. J Clin Oncol. 2013;31(16):2029-36.

14. Aagaard K, Ma J, Antony KM, Ganu R, Petrosino J, Versalovic J. The placenta harbors a unique microbiome. Science translational medicine. 2014;6(237):237ra65.

15. Parker CC, James ND, Brawley CD, Clarke NW, Hoyle AP, Ali A, et al. Radiotherapy to the primary tumour for newly diagnosed, metastatic prostate cancer (STAMPEDE): a randomised controlled phase 3 trial. Lancet. 2018;392(10162):2353-66.

16. Burdett S, Boeve LM, Ingleby FC, Fisher DJ, Rydzewska LH, Vale CL, et al. Prostate Radiotherapy for Metastatic Hormone-sensitive Prostate Cancer: A STOPCAP Systematic Review and Meta-analysis. European urology. 2019;76(1):115-24.

17. Sooriakumaran $P$. Testing radical prostatectomy in men with prostate cancer and oligometastases to the bone: a randomized controlled feasibility trial. BJU Int. 2017;120(5B):E8-E20.

18. Rexer H. [Metastatic, hormone-naive prostate cancer interventional study : Multicenter, prospective, randomized study to evaluate the effect of standard drug therapy with or without radical prostatectomy in patients with limited bone metastasized prostate cancer (G-RAMPP - the AUO AP 75/13 study)]. Urologe A. 2015;54(11):1613-6.

19. Tannock IF, de Wit R, Berry WR, Horti J, Pluzanska A, Chi KN, et al. Docetaxel plus prednisone or mitoxantrone plus prednisone for advanced prostate cancer. The New England journal of medicine. 2004;351(15):1502-12.

20. James ND, Sydes MR, Clarke NW, Mason MD, Dearnaley DP, Spears MR, et al. Addition of docetaxel, zoledronic acid, or both to first-line long-term hormone therapy in prostate cancer (STAMPEDE): survival results from an adaptive, multiarm, multistage, platform randomised controlled trial. Lancet. 2016;387(10024):1163-77.

21. Gravis G, Fizazi K, Joly F, Oudard S, Priou F, Esterni B, et al. Androgen-deprivation therapy alone or with docetaxel in non-castrate metastatic prostate cancer (GETUG-AFU 15): a randomised, open-label, phase 3 trial. Lancet Oncol. 2013;14(2):149-58.

22. Kyriakopoulos CE, Chen YH, Carducci MA, Liu G, Jarrard DF, Hahn NM, et al. Chemohormonal Therapy in Metastatic Hormone-Sensitive Prostate Cancer: Long-Term Survival Analysis of the Randomized Phase III E3805 CHAARTED Trial. J Clin Oncol. 2018;36(11):1080-7.

23. Gravis G, Boher JM, Joly F, Soulie M, Albiges L, Priou F, et al. Androgen Deprivation Therapy (ADT) Plus Docetaxel Versus ADT Alone in Metastatic Non castrate Prostate Cancer: Impact of Metastatic Burden and Long-term Survival Analysis of the Randomized Phase 3 GETUG-AFU15 Trial. European urology. 2016;70(2):256-62.

24. Gravis G, Boher JM, Chen YH, Liu G, Fizazi K, Carducci MA, et al. Burden of Metastatic Castrate Naive Prostate Cancer Patients, to Identify Men More Likely to Benefit from Early Docetaxel: Further Analyses of CHAARTED and GETUG-AFU15 Studies. European urology. 2018;73(6):847-55. 
25. Clarke NW, Ali A, Ingleby FC, Hoyle A, Amos CL, Attard G, et al. Addition of docetaxel to hormonal therapy in low- and high-burden metastatic hormone sensitive prostate cancer: long-term survival results from the STAMPEDE trial. Ann Oncol. 2019.

26. Ryan CJ, Smith MR, Fizazi K, Saad F, Mulders PF, Sternberg CN, et al. Abiraterone acetate plus prednisone versus placebo plus prednisone in chemotherapy-naive men with metastatic castration-resistant prostate cancer (COU-AA-302): final overall survival analysis of a randomised, double-blind, placebo-controlled phase 3 study. Lancet Oncol. 2015;16(2):152-60.

27. de Bono JS, Logothetis CJ, Molina A, Fizazi K, North S, Chu L, et al. Abiraterone and increased survival in metastatic prostate cancer. The New England journal of medicine. 2011;364(21):1995-2005.

28. James ND, de Bono JS, Spears MR, Clarke NW, Mason MD, Dearnaley DP, et al. Abiraterone for Prostate Cancer Not Previously Treated with Hormone Therapy. The New England journal of medicine. 2017;377(4):338-51.

29. Fizazi K, Tran N, Fein L, Matsubara N, Rodriguez-Antolin A, Alekseev BY, et al. Abiraterone plus Prednisone in Metastatic, Castration-Sensitive Prostate Cancer. The New England journal of medicine. 2017;377(4):352-60.

30. Hoyle AP, Ali A, James ND, Cook A, Parker CC, de Bono JS, et al. Abiraterone in "High-" and "Low-risk" Metastatic Hormone-sensitive Prostate Cancer. European urology. 2019.

31. Beer TM, Armstrong AJ, Rathkopf DE, Loriot Y, Sternberg CN, Higano CS, et al. Enzalutamide in metastatic prostate cancer before chemotherapy. The New England journal of medicine. 2014;371(5):424-33.

32. Scher HI, Fizazi K, Saad F, Taplin ME, Sternberg CN, Miller K, et al. Increased survival with enzalutamide in prostate cancer after chemotherapy. The New England journal of medicine. 2012;367(13):1187-97.

33. Davis ID, Martin AJ, Stockler MR, Begbie S, Chi KN, Chowdhury S, et al. Enzalutamide with Standard First-Line Therapy in Metastatic Prostate Cancer. The New England journal of medicine. 2019;381(2):121-31.

34. Armstrong AJ, Szmulewitz RZ, Petrylak DP, Holzbeierlein J, Villers A, Azad A, et al. ARCHES: A Randomized, Phase III Study of Androgen Deprivation Therapy With Enzalutamide or Placebo in Men With Metastatic Hormone-Sensitive Prostate Cancer. J Clin Oncol. 2019:JCO1900799.

35. Chi KN, Agarwal N, Bjartell A, Chung BH, Pereira de Santana Gomes AJ, Given R, et al. Apalutamide for Metastatic, Castration-Sensitive Prostate Cancer. The New England journal of medicine. 2019;381(1):13-24.

36. Eriksson S, Eriksson A, Stege R, Carlstrom K. Bone mineral density in patients with prostatic cancer treated with orchidectomy and with estrogens. Calcif Tissue Int. 1995;57(2):97-9. 
37. Smith MR, Eastham J, Gleason DM, Shasha D, Tchekmedyian S, Zinner N. Randomized controlled trial of zoledronic acid to prevent bone loss in men receiving androgen deprivation therapy for nonmetastatic prostate cancer. J Urol. 2003;169(6):2008-12.

38. Greenspan SL, Nelson JB, Trump DL, Resnick NM. Effect of once-weekly oral alendronate on bone loss in men receiving androgen deprivation therapy for prostate cancer: a randomized trial. Ann Intern Med. 2007;146(6):416-24.

39. Egerdie B, Saad F. Bone health in the prostate cancer patient receiving androgen deprivation therapy: a review of present and future management options. Can Urol Assoc J. 2010;4(2):129-35.

40. Smith MR, Egerdie B, Hernandez Toriz N, Feldman R, Tammela TL, Saad F, et al. Denosumab in men receiving androgen-deprivation therapy for prostate cancer. The New England journal of medicine. 2009;361(8):745-55.

41. Bone HG, Wagman RB, Brandi ML, Brown JP, Chapurlat R, Cummings SR, et al. 10 years of denosumab treatment in postmenopausal women with osteoporosis: results from the phase 3 randomised FREEDOM trial and open-label extension. Lancet Diabetes Endocrinol. 2017;5(7):513-23.

42. Bai H, Jing D, Guo A, Yin S. Randomized controlled trial of zoledronic acid for treatment of osteoporosis in women. J Int Med Res. 2013;41(3):697-704. 